OPEN ACCESS

Edited by:

Marzia Duse,

Sapienza University of Rome, Italy

Reviewed by:

Mauro Bacci,

University of Perugia, Italy Isabella Aquila

University of Catanzaro, Italy

*Correspondence:

Luigi Cipolloni

luigi.cipolloni@unifg.it

tThese authors have contributed equally to this work

¥These authors share last authorship

Specialty section:

This article was submitted to General Pediatrics and Pediatric

Emergency Care,

a section of the journal

Frontiers in Pediatrics

Received: 14 November 2019

Accepted: 15 April 2020

Published: 27 May 2020

Citation:

Bertozzi G, Maglietta F, Baldari B,

Besi L, Torsello A, Di Gioia CRT,

Sessa F, Aromatario M and Cipolloni L

(2020) Mistrial or Misdiagnosis: The

Importance of Autopsy and

Histopathological Examination in

Cases of Sudden Infant

Bronchiolitis-Related Death

Front. Pediatr. 8:229.

doi: 10.3389/fped.2020.00229

\section{Mistrial or Misdiagnosis: The Importance of Autopsy and Histopathological Examination in Cases of Sudden Infant Bronchiolitis-Related Death}

\author{
Giuseppe Bertozzi ${ }^{1 \dagger}$, Francesca Maglietta ${ }^{1 \dagger}$, Benedetta Baldari ${ }^{2}$, Livia Besi ${ }^{2}$, \\ Alessandra Torsello', Cira Rosaria Tiziana Di Gioia ${ }^{3}$, Francesco Sessa ${ }^{1}$, \\ Mariarosaria Aromatario ${ }^{2 \neq}$ and Luigi Cipolloni ${ }^{1 * \neq}$
}

\begin{abstract}
${ }^{1}$ Department of Clinical and Experimental Medicine, Section of Legal Medicine, University of Foggia, Foggia, Italy, ${ }^{2}$ Department of Anatomical, Histological, Forensic and Orthopedic Sciences, Sapienza University of Rome, Rome, Italy, ${ }^{3}$ Department of Radiological, Oncological and Pathological Sciences, Sapienza University of Rome, Rome, Italy
\end{abstract}

Pediatrics, among all the branches of medicine, is a sector not particularly affected by a high number of claims. Nevertheless, the economic value of the compensation is significantly high, for example, in cases of children who suffered multiple disabilities following perinatal lesions with a long life expectancy. In Italy, most of the claims for compensation concern surgical pathologies and infections. Among these latter, the dominant role is taken by respiratory tract infections. In this context, the purpose of this manuscript is to present a case series of infant deaths in different emergency-related facilities (ambulances, emergency rooms) denounced by relatives. Following these complaints, the autopsy was performed, and subsequent histological examinations revealed the presence of typical and pathognomonic histological findings of acute viral bronchiolitis, whose morphological appearance is poorly reported in the literature. The analysis of these cases made it possible to highlight the following conclusions: the main problems in diagnosing sudden death causes, especially in childhood, are the rapidity of death and the scarce correlation between the preexistent diseases and of the cause of death itself. For all these reasons, the autopsy, either clinical or medicolegal, is mandatory in cases of sudden unexpected infant death to manage claim requests because only the histological examinations performed on samples collected during the autopsy can reveal the real cause of death.

Keywords: sudden unexpected infant deaths (SUIDs), sudden infant death, autopsy, immunohistochemistry, acute viral bronchiolitis

\section{INTRODUCTION}

Pediatrics, among the branches of medicine, is not particularly affected by a high number of claims. Nevertheless, the economic value of compensation is significantly high, for example, in cases of children who suffered multiple disabilities following perinatal lesions with a long life expectancy. According to Carroll and Buddenbaum (1) and Moriani et al. (2), examining the data collected 
by an association of several American insurance companies (Physician Insurers Association of America), it was found that only $28 \%$ of the cases resulted in compensation; among these, in cases where no damages had been paid, the average cost per the only defense was $\$ 28,779$, while it results in $\$ 67,502$ for paid claims (3). The medical diagnoses, most commonly involved in civilian pediatric trials in the United States, were brain damage (average damages $\$ 440,379)$ and meningitis $(\$ 437,423$ ). Respiratory problems in newborns account for $\$ 270,607$. In Italy, data from the insurance company CARIGE Spa, in the period 2005-2012, excluding neonatology, highlighted how the main pathologies for which legal action was referred to surgery (gastrointestinal and testicular) and infections (more respiratory ones). Moreover, the data on litigation have also shown a different stratification of the number of requests for compensation, which were greater in the north and minors in central Italy, mostly involving the public health system (4). According to the Italian study performed during the 2005-2010 period, neonatology has also shown an overlapping geographical stratification, with the greatest interest for the public sector. The claims for damages following death, concerning the neonatal intensive care unit (NICU), mainly concern respiratory diseases (30.7\% of cases) $(5,6)$. Both in the pediatric population in general, but especially in the neonatological one, the dominant role is taken by the respiratory tract infections.

In this context, the purpose of these case series is to demonstrate how the identification of the correct cause of death in the sudden unexpected infant deaths (SUIDs) allowed evaluating the absence of medical liability. Particularly, the definition of gold standard methods in similar cases could be considered very important to avoid the compensation in unjustified claim requests.

\section{CASE SERIES}

All procedures performed in the study were in accordance with the ethical standards of the institution and with the 1964 Helsinki Declaration and its later amendments or comparable ethical standards. Written informed consent was obtained from the first-degree relatives.

\section{Case 1}

A 10-month-old male infant died during Emergency Medical Services (EMS) transport to the hospital. When parents had been asked for any modification in their child habits, a mild "rhinitis" for a few days was told. For this reason, they went to their trusted pediatrician 2 days earlier, who suggested saline nasal rinses and a short turn check. The medical examiner documented no relevant external sign to explain death. Therefore, the parents sued the pediatrician for both penal and civil liability. During the forensic autopsy, the macroscopic examination was unremarkable except for mild edema affecting both lungs. On the contrary, histological examination showed in both lungs a diffuse transmural inflammation in the bronchiolar wall. Other tissue sections showed chronic inflammation, and bronchiolar wall fibrosis primarily restricted to bronchioles (Figure 1).

\section{Case 2}

An ambulance was called for a 9-month-old female infant, who lived in a nomad camp; her parents referred that suddenly she did not respond to external stimuli. Relatives did not refer to any symptoms neither clinical signs in the previous days. During the resuscitation maneuvers in place, the infant died. Thus, the prosecutor ordered the autopsy for alleged medical liability; her parents demanded the civil compensation to the local health insurance, thinking that during the ambulance transportation, there was medical responsibility. The macroscopic examination, both at the external corpse and internal organs, only showed severe pulmonary edema. The histology was characterized by lymphocytic infiltration of the bronchioles (Figure 2).

\section{Case 3}

An 18-month-old female infant was admitted to the emergency room of a pediatric hospital for severe cough and pharyngitis; she died after a few hours. Symptoms onset occurred the day before hospitalization. She was a preterm infant (29.3 weeks, birth weight $1,400 \mathrm{~g}$ ) who suffered from severe respiratory distress at birth (Apgar score $1^{\prime}=4$ ) and needed a long period of hospitalization. After discharge, she showed neurodevelopmental impairment; moreover, a month before death, she suffered from many viral infective pathologies such as influenza and

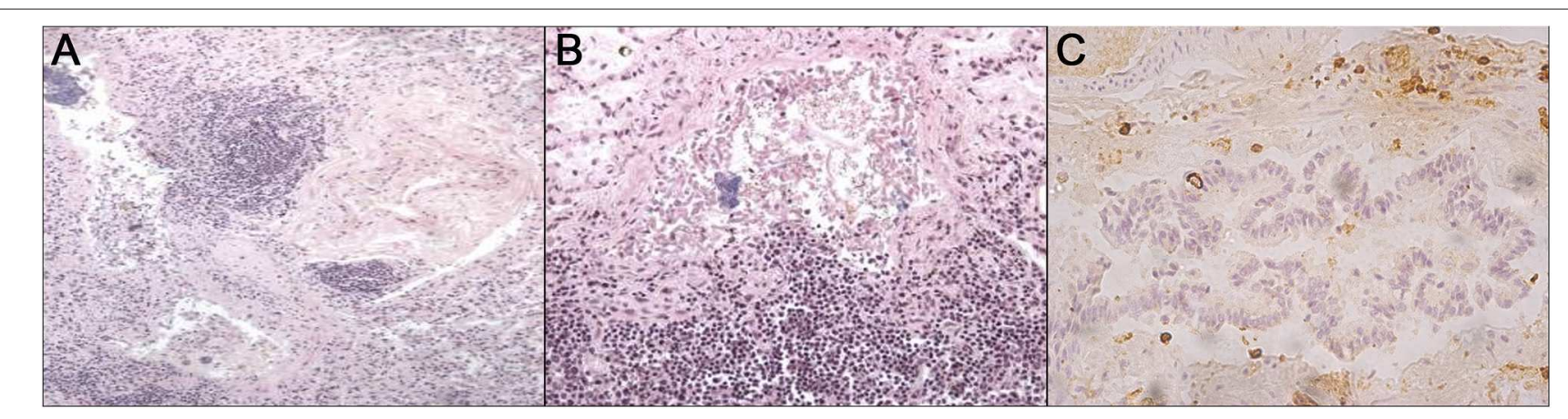

FIGURE 1 | From left to right: (A) HandE staining let a leukocyte bronchial and peribronchial infiltrate be noticed; (B) HandE staining shows the bronchial walls with epithelial cells fallen into the lumen which appears filled by abundant amorphous eosinophilic material in which leukocytes can be detected; (C) immunohistochemical staining with anti-CD45 reveals peribronchial positivity. 
mononucleosis: all pathologies were successfully treated with standard pharmacological therapies. In this case, the judicial authority disposed of the forensic examination, suspecting medical liability to clarify penal and civil aspects: indeed, at the time of death, a claim for damages has been made to the hospital by the family of the patient. The autopsy showed congestion of tracheal and bronchial mucosa. At histological examination, focal edema and diffuse congestion of both lungs, acute emphysema, and peribronchial and intrabronchial wall leukocyte infiltrates were found; the same results involved nearby septal vessels (Figure 3).

\section{MATERIALS AND METHODS}

The method used in these cases, as in all cases of sudden death, consists of a rigorous and multidisciplinary methodological approach $(7,8)$ :

- anamnestic collection and clinical findings: the clinical symptomatology presented by the subject before his death or in close chronological concurrence, clinical history of the case, and previous medical records;

- anatomical evidence: a macroscopic examination of all organs, their weight, consistency, and color at the autopsy, such as the appearance of fluids (blood, urine, vitreous humor). histomorphological examination $\mathrm{H} \& \mathrm{E}$ of the organ samples to study any alteration due to pathological condition;

immunohistochemistry: to evaluate, in particular, the presence and the location of the main white blood cells via antibody anti-leukocyte common antigen (CD45).

The adherence to this diagnostic procedure not only allows you to check and evaluate a larger quantity of data but allows a complete evaluation on all fronts, from the study of which can confirm the suspicion and/or an unexpected result but crucial for investigations.

\section{DISCUSSION}

In the discussed cases, following both the autoptic and especially the microscopic examination, the cause of death was identified in all investigated cases: a rapidly progressive acute bronchiolitis was ascertained. These findings allowed exonerating doctors from any penal liability. The bronchiolitis was defined in 2006, from a collaboration between the American Academy of Pediatrics (AAP) and the European Respiratory Society (ERS), as "a constellation of clinical symptoms and signs including a viral upper respiratory prodrome followed by increased respiratory effort and wheezing in children $<2$ years of age" (9). Acute viral bronchiolitis (AVB) is a lower respiratory tract infective disorder, typically affecting infants $<2$ years old (90\% of cases).



FIGURE 2 | From left to right: (A) the not significant macroscopic study; (B) histological study; (C) immunohistochemistry shows the positivity to anti-CD45 staining. HandE staining shows considerable thickening of the septal structures due to the presence of abundant cellularity, mainly consisting of plasma cells and small lymphocytes. The alveolar spaces are atelectatic in some fields; in others, they show amorphous eosinophilic material inside. In many fields, bronchial and peribronchial infiltrates, consisting of lymphocytes and plasma cells, cover the bronchial structure itself.



FIGURE 3 | (A-C) Immunohistochemical staining CD45-positive showing peribronchial and intrabronchial wall leukocyte infiltrates. 
Respiratory syncytial virus (RSV) is involved in up to $70 \%$ of cases, followed by rhinovirus (up to 25\%); the remaining cases are related to coronavirus, adenovirus, influenza, and parainfluenza virus, and human metapneumovirus. Coinfections are common (10). However, the seasonality of bronchiolitis, generally more frequently found during the winter months, coincides with the seasonal pattern of RSV diffusion (11).

The infection starts in the upper respiratory tract, spreading to the lower airways in a few days. The bronchiolar damage is determined by the direct action of the virus on the epithelium of the same tract; alternatively, it was indirectly immunemediated, and it was characterized by a peribronchial infiltration of white blood cell types, mainly mononuclear cells, with edema of the submucosa and adventitia (9). The pathophysiological continuation is caused by a mixture of edema, increased production of mucus, and progressive damage of the epithelium even to necrosis, which determines obstruction of the airflow, entrapment of distal air, atelectasis, and alteration of the ventilation/perfusion. The results are hypoxemia and increased respiratory work, which in turn worsens hypoxemia (9).

The most important extrapulmonary symptoms involve the brain (apnea, epileptic status) and heart (ventricular tachycardia, ventricular fibrillation, cardiogenic shock, complete heart block, and pericardial tamponade) and are common in children with severe infections (12).

The most dreadful complications of bronchiolitis are central apnea, a respiratory pause with bradycardia, cyanosis, pallor, and hypotonia that often requires hospitalization (13). Bronchiolitis represents a disease with high morbidity but low mortality. Death from respiratory failure in bronchiolitis is rare and varies from deaths from 2.9:100,000 in the UK to 5.3:100,000 in the US, for children under 12 months, with a relationship that goes hand in hand, reducing itself to the improvement of good intensive practices $(9,14-16)$.

In all these cases, in the absence of the clinical-anamnestic data that can guide the clinical diagnosis, the external examination data and the autopsy macroscopic data could point toward a diagnosis of SUID. In the case of Özdemir et al. (17), in fact, on the totality of the cases of malpractice claims, $57.5 \%$ of the children had died and $59.3 \%$ were subjected to autopsy. In these cases, the causes of death reported before and after the autopsies were different in $68 \%$, and the medical staff was found to be responsible for $46.1 \%$ of the claims.

Therefore, the determination of the exact cause of death assumes fundamental importance to ascertain the causal link of any conduct of both health facilities and individual professionals in determining death (18-20). This allows not only a measurement of the quality of care provided by promoting public trust for the health system but also as a measure of clinical governance; moreover, it is possible to better manage the medicolegal disputes as a guarantee of ascertaining the truth. Indeed, in Italy, the data on the frequency of adverse events (AEs), preventable adverse events (PAEs), and negligent adverse events (NAEs) are available; nevertheless, data about malpractice claims are not available both under the penal and civil points of view. Furthermore, the epidemiological purposes cannot be forgotten, considering that it is the only reliable method of data collection. Indeed, a complete methodological approach, integrating clinical data, autopsy, and histological findings could be considered the best way to solve similar cases. In fact, in the reported case studies, histopathologic diagnostics identified pathognomonic signs of acute bronchiolitis characterized by edema, congestion, leukocytic infiltration in the bronchiolar wall, leukocytes in the peribronchial interstitial pulmonary space, allowing the identification of the exact cause of death. Therefore, these pieces of evidence have allowed excluding the medical responsibility in the reported cases, demonstrating that there are events not related to the supplied health care.

\section{CONCLUSION}

The analysis of the presented cases shows that the autopsy is mandatory in SUID occurrence, in which the absence of anamnestic data and/or acute clinical signs does not allow to identify the cause of death. Hypothesizing medical negligence in each case, the autopsy was performed following the judicial appointment after the relative's complaint.

The subsequent histological examinations revealed the presence of typical and pathognomonic histological findings of $\mathrm{AVB}$, whose morphological appearance is poorly described in the literature. Only the postmortem examinations have allowed excluding medical liability and therefore the compensation for damage.

In light of these findings, it could be considered essential an accurate evaluation of similar cases, collecting all data to avoid compensation in unjustified claims made against the hospital. In this way, it is possible to contain the hospital costs related to this kind of accident. For all these reasons, the autopsy combined with the subsequent examination represents a gold standard method to identify the absence of the hospital's responsibility in SUID cases.

\section{DATA AVAILABILITY STATEMENT}

All datasets generated for this study are included in the article/supplementary material.

\section{ETHICS STATEMENT}

Written informed consent was obtained from the first-degree relatives for the publication of this case report.

\section{AUTHOR CONTRIBUTIONS}

GB, FM, MA, and LC contributed to the conception of the study and wrote the manuscript. FS, BB, LB, AT, and CD contributed significantly to literature review and manuscript preparation. GB, FM, FS, BB, LB, AT, CD, MA, and LC helped perform the analysis with constructive discussions and approved the final version. 


\section{REFERENCES}

1. Carroll AE, Buddenbaum JL. Malpractice claims involving pediatricians: epidemiology and etiology. Pediatrics. (2007) 120:10-7. doi: 10.1542/peds.2006-3618

2. Moriani S, Cecchi R, Cipolloni L. Suicide by sharp instruments: a case of harakiri. Int J Legal Med. (1996) 108:219-20. doi: 10.1007/BF01369796

3. McAbee GN, Donn SM, Mendelson RA, McDonnell WM, Gonzalez JL, Ake JK. Medical diagnoses commonly associated with pediatric malpractice lawsuits in the united states. Pediatrics. (2008) 122:e1282-6. doi: 10.1542/peds.2008-1594

4. Agostiniani R, Correra A, D’Agostino P, D’Aloja E, Greco L, Tagliabue P, et al. Pediatric claims in Italy during a 8-years survey. Ital J Pediatr. (2014) 40:A87. doi: 10.1186/1824-7288-40-S1-A87

5. Fanos V, Tagliabue P, Greco L, Agostiniani R, Carbone MT, D’Agostino P, et al. Neonatal malpractice claims in Italy: How big is the problem and which are the causes? J Matern Neonatal Med. (2012) 25:493-7. doi: 10.3109/14767058.2011.622004

6. Vullo A, Panebianco V, Cannavale G, Aromatario M, Cipolloni L, Frati P, et al. Post-mortem magnetic resonance foetal imaging: a study of morphological correlation with conventional autopsy and histopathological findings. Radiol Medica. (2016) 121:847-56. doi: 10.1007/s11547- 016-0672-z

7. Ferrara M, Sessa F, Rendine M, Spagnolo L, De Simone S, Riezzo I, et al. A multidisciplinary approach is mandatory to solve complex crimes: a case report. Egypt J Forensic Sci. (2019) 9:11. doi: 10.1186/s41935-0190116-8

8. Maglietta F, Sessa F, Albano GD, Bertozzi G, Giugliano P, Vacchiano G, et al. Italian Mafia: a focus on Apulia Mafia with A literature review. Acta Medica Mediterr. (2017) 2017:947-52. doi: 10.19193/0393-6384_2017_6_149

9. Øymar K, Skjerven HO, Mikalsen IB. Acute bronchiolitis in infants, a review. Scand J Trauma Resusc Emerg Med. (2014) 22:23. doi: 10.1186/1757-7241-22-23

10. Mansbach JM, Piedra PA, Teach SJ, Sullivan AF, Forgey T, Clark S, et al. Prospective multicenter study of viral etiology and hospital length of stay in children with severe bronchiolitis. Arch Pediatr Adolesc Med. (2012) 166:7006. doi: 10.1001/archpediatrics.2011.1669

11. Horn SD, Smout RJ, King J. Effect of prematurity on respiratory syncytial virus hospital resource use and outcomes. J Pediatr. (2003) 143:133-41. doi: 10.1067/s0022-3476(03)00509-2

12. Eisenhut M. Extrapulmonary manifestations of severe respiratory syncytial virus infection - A systematic review. Crit Care. (2006) 10:R107. doi: $10.1186 / \mathrm{cc} 4984$
13. Ricart S, Rovira N, Garcia-Garcia JJ, Pumarola T, Pons M, Muñoz-Almagro C, et al. Frequency of apnea and respiratory viruses in infants with bronchiolitis. Pediatr Infect Dis J. (2014) 33:988-90. doi: 10.1097/INF.0000000000000365

14. Ecochard-Dugelay E, Beliah M, Perreaux F, de Laveaucoupet J, Bouyer J, Epaud R, et al. Clinical predictors of radiographic abnormalities among infants with bronchiolitis in a paediatric emergency department. BMC Pediatr. (2014) 14:143. doi: 10.1186/1471-2431-14-143

15. Florin TA, Byczkowski T, Ruddy RM, Zorc JJ, Test M, Shah SS. Variation in the management of infants hospitalized for bronchiolitis persists after the 2006 American Academy of Pediatrics bronchiolitis guidelines. J Pediatr. (2014) 165:786-92.e1. doi: 10.1016/j.jpeds.2014.05.057

16. Holman RC, Shay DK, Curns AT, Lingappa JR, Anderson LJ. Risk factors for bronchiolitis-associated deaths among infants in the United States. Pediatr Infect Dis J. (2003) 2003:22. doi: 10.1097/01.inf.0000069765.43405.3b

17. Özdemir MH, Ergönen TA, Can IÖ. Medical malpractice claims involving children. Forensic Sci Int. (2009) 191:80-5. doi: 10.1016/j.forsciint.2009.06.014

18. Albano GD, Bertozzi G, Maglietta F, Montana A, Di Mizio G, Esposito M, et al. Medical records quality as prevention tool for Healthcare-Associated Infections (HAIs) related litigation: a case series. Curr Pharm Biotechnol. (2019) 20:653-7. doi: 10.2174/1389201020666190408102221

19. Borro M, Gentile G, Cipolloni L, Foldes-Papp Z, Frati P, Santurro A, et al. Personalised healthcare: the DiMA clinical model. Curr Pharm Biotechnol. (2017) 18:242-52. doi: 10.2174/1389201018666170208 125131

20. Di Paolo M, Papi L, Malacarne P, Gori F, Turillazzi E. Healthcare-associated infections: not only a clinical burden, but a forensic point of view. Curr Pharm Biotechnol. (2019) 20:658-64. doi: 10.2174/1389201020666190618122649

Conflict of Interest: The authors declare that the research was conducted in the absence of any commercial or financial relationships that could be construed as a potential conflict of interest.

The handling editor declared a shared affiliation, though no other collaboration, with the authors GB, FM, BB, LB, AT, CD, FS, MA, and LC.

Copyright (๔) 2020 Bertozzi, Maglietta, Baldari, Besi, Torsello, Di Gioia, Sessa, Aromatario and Cipolloni. This is an open-access article distributed under the terms of the Creative Commons Attribution License (CC BY). The use, distribution or reproduction in other forums is permitted, provided the original author(s) and the copyright owner(s) are credited and that the original publication in this journal is cited, in accordance with accepted academic practice. No use, distribution or reproduction is permitted which does not comply with these terms. 